\title{
Entre la neuroética y la neuroeducación: las fronteras de las neurociencias sociales
}

\author{
Between neuroethics and neuroeducation: the frontiers \\ of social neurosciences
}

El presente monográfico de Recerca. Revista de Pensament i Anàlisi, titulado "Neuroética y neuroeducación: repensando la relación entre las neurociencias y las ciencias sociales», está formado por ocho artículos de investigación y cuatro recensiones, a través de los cuales se estudian de forma actualizada las relaciones entre las neurociencias y las ciencias sociales. Dentro de las múltiples relaciones que se generan en la conjunción interdisciplinaria de estas dos materias, se destacan especialmente la neuroética y la neuroeducación; sin embargo, temáticamente se acogen perspectivas como la neuropolítica, o la relación entre emoción y razón desde las neurociencias, entre otras.

No cabe duda de que, tras quince años de su nacimiento, la neuroética se ha desarrollado ampliamente como disciplina. Ya no solo se la concibe como una rama de la bioética (Glannon, 2006), sino que desde su vertiente como neuroética fundamental o teórica (Evers, 2010; Levy, 2007), o «neurociencia de la ética» (Roskies, 2002), ha ganado una gran pertinencia normativa y metodológica debido al uso de técnicas neurocientíficas como la fMRI o la electroencefalografía entre otras (Farah, 2011). Esto podría ser en principio

\footnotetext{
* Este monográfico se inserta dentro de los objetivos del proyecto de la subvención para la contratación de personal investigador en fase posdoctoral [APOSTD/2017/003], concedida por la Consellería de Educación, Cultura y Deporte de la Generalitat Valenciana, y el Fondo Social Europeo. Además, esta contribución se enmarca en los objetivos concretos del proyecto de investigación de Ministerio de Economía y Competitividad titulado: «Neuroeducación moral para las éticas aplicadas» [FFI201676753-C2-2-P].

* Este monográfico se enmarca dentro de los objetivos concretos del proyecto de investigación del Ministerio de Economía y Competitividad titulado: «Neuroeducación moral, democracia deliberativa y políticas de desarrollo humano sostenible» [FFI2016-76753-C2-1-P].
} 
muy beneficioso para la interdisciplinariedad científica; no obstante, en numerosas ocasiones se ha malinterpretado esta relación, intentándose sustituir el conocimiento de la filosofía y las ciencias sociales por el conocimiento empírico natural.

En otros trabajos previos de Recerca. Revista de Pensament i Anàlisi, se ha reflexionado sobre los límites de las neurociencias para alcanzar, por ellas mismas y de una manera total, la explicación de las esferas sociales y del comportamiento humano. En efecto, en el monográfico número 13, titulado «Retos actuales de la neuroética» (Conill \& Pérez-Zafrilla, 2013), ya se hizo hincapié en la cuestión de la metodología de las neurociencias sociales en este sentido y en las potencialidades de sus nuevos descubrimientos, como la mejora cerebral y la aceptabilidad ética de su aplicación.

La neuroética, como temática, se ha tratado también en el artículo de C. Ortiz de Landázuri (2016): «El error neurocientífico de Descartes, entre Spinoza y Aquinas. El debate entre Damasio y Stump sobre el carácter eliminativo o vitalista del materialismo en la neuroética, neuropolítica y neuroeconomía», perteneciente al monográfico número 18 de la revista. En este artículo se analizaba el dualismo cartesiano respecto a la mente y al cerebro, y se aportaba como posible solución más allá del monismo, un modelo dualista vitalista de integración jerarquizada e interactiva entre ambos.

Pero la neuroética no es la única que se ha desarrollado en estos años, sino que paralelamente a ella han surgido otras disciplinas como la neuroeconomía (Conill, 2012) la neuropolítica (García-Marzá, 2012) el neuromarketing (Feenstra \& Pallarés-Domínguez, 2017) o la neuroeducación (Codina, 2015). Todo un conjunto de nuevos campos de estudio en los que se hace imprescindible una reflexión crítica sobre sus planteamientos metodológicos y una mayor perspectiva ética (Cortina, 2012; García-Marzá \& Feenstra, 2013). Esta última, la neuroeducación, como campo de estudio propio, ha contribuido al proceso de enseñanza-aprendizaje de una forma general, especialmente en su intento de entender cómo aprende el cerebro humano y analizar presupuestos pedagógicos a la luz de las neurociencias (Ansari et al., 2011; Campbell, 2011). Sin embargo, en este monográfico se cuestiona particularmente la relación entre la neuroética y la neuroeducación, es decir, si sería posible educar moralmente teniendo en cuenta los descubrimientos de las neurociencias. En otras palabras, si facultades sociales como la empatía, las predisposiciones y emociones asociadas a la prosocialidad y el correcto ejercicio de facultades y funciones ejecutivas como la razón o la memoria pueden ser educadas y posibilitar así el desarrollo moral del individuo. 
Pero más allá de las posibilidades que podría ofrecer una verdadera neuroeducación moral -por ahora una disciplina en construcción-, este monográfico analiza también otras cuestiones metodológicas más amplias que afectan a todas las neurociencias sociales en general, como el naturalismo, el monismo metodológico o la interdisciplinariedad científica. Desde una perspectiva mayoritariamente teórica y crítica, los artículos del presente monográfico han contribuido a este objetivo.

En primer lugar, el artículo de Òscar Llorens nos remite directamente a uno de los principales problemas de las neurociencias sociales para lograr una interdisciplinariedad científica. Por un lado, al extremo de lo que él considera «neurofilia», es decir, el optimismo prometedor de la neurociencia con respecto a las ciencias humanas y sociales, y, por otro lado, al neuroescepticismo que muchos filósofos han desarrollado al comprobar que solamente son intentos de substituir el saber social por el saber natural. Haciendo un repaso propio de la historia y la filosofía de la ciencia, cuestiona las ventajas e inconvenientes del dualismo y del naturalismo como soluciones a la naturaleza de la consciencia humana. Se destaca la amplia bibliografía analizada, que ayuda sin duda al lector a situarse tanto en los antecedentes inmediatos como en los problemas más actuales de la neurociencia en relación con las ciencias sociales.

En segundo lugar, el artículo de Paula Castelli destaca la cuestión central del monográfico, es decir, el modelo de relación interdisciplinaria que requeriría la neuroética como una disciplina en gran parte filosófica, y aún más si se pretende alcanzar en algún momento una neuroeducación moral basada en ella. Para ello, la profesora de la Universidad de Buenos Aires, analiza dos niveles diferentes de relación entre los argumentos filosóficos y las evidencias empíricas en la neuroética desde una perspectiva teórica - neurociencia de la ética en este caso (Roskies, 2002). Finalmente, descarta el naturalismo como opción, y opta por tener en cuenta la evidencia neurocientífica como explicación parcial pero no total del comportamiento moral.

En tercer lugar, el artículo de Javier Gracia se centra en la neurociencia educativa -educational neuroscience- y en él apuesta por la ética como una disciplina clave en el renombrado ya trinomio 'educación-neurociencia-psicología' que forma la neuroeducación. Teniendo en cuenta las aportaciones de la neuroética filosófica y, al igual que el artículo anterior, descartando el naturalismo como metodología viable, el profesor de la Universitat de València pretende expandir la neuroeducación a un nuevo enfoque que no se reduzca meramente a la adaptación. Es especialmente interesante, en este sentido, el análisis que realiza sobre cómo entender las evidencias neurocientíficas en el campo de la enseñanza, así como la revisión crítica de autores ya clásicos de 
la neuroeducación como P. H. Jones (2011), D. Ansari (2005) o U. Goswami (2004), y de otros más novedosos como J. S. Bowser (2016).

En cuarto lugar, el artículo de Miguel Grijalba supone un análisis exhaustivo de la obra de A. Damasio en relación con una de sus más conocidas obras, como es El error de Descartes (2003). El profesor de filosofía de la UNED propone que el error, en este caso, es de Damasio y no de Descartes. Por un lado, su crítica destaca que Damasio se ciñe solamente al nivel antropológico, olvidando la cuestión ontológica sobre el conocimiento, lo que le lleva a cometer la falacia de la objetividad y también una naturalización de la mente. Por otro lado, pone de manifiesto que, a pesar de que el propio Damasio reconoce que no sabemos realmente cómo el cerebro produce la mente - por lo que termina optando por un emergentismo débil-, aun así, en muchas ocasiones identifica las tesis cientificistas con las tesis científicas. Sin duda, uno de los puntos más destacables de este artículo es la defensa de Descartes y la argumentación para rechazar el dualismo absoluto basándose en la obra Las pasiones del alma (2005).

En quinto lugar, el artículo de Pedro Jesús Pérez Zafrilla aborda la relación entre las neurociencias y las ciencias sociales desde la neuropolítica, centrándose en la cuestión de la traslación terminológica entre ambas. Concretamente, analiza la noción de «marco mental» desde la obra de G. Lakoff (2009) y J. Haidt (2012), comparándola con la noción filosófica de «marco referencial» de Ch. Taylor (1996). Destaca especialmente cómo una parte de la política se enfoca a la manipulación emocional mediante la generación de intuiciones y la activación de marcos mentales. Una democracia a la altura de nuestros tiempos no puede, en opinión del profesor de la Universitat de València, asentarse sobre estos presupuestos, sino que, teniendo en cuenta el mundo moral, debe argumentar también mediante razones sobre la deliberación pública.

En sexto lugar, y siguiendo con el tema de las emociones en el campo de la política, el artículo de José Manuel Panea Márquez analiza de qué forma las emociones se pueden utilizar precisamente para lo contrario de lo que se criticaba en el artículo anterior. Es decir, cómo las emociones pueden contribuir a reforzar la vida democrática. Para ello, el profesor de la Universidad de Sevilla, se centra en la figura de M. C. Nussbaum (2002), especialmente en su tratamiento de las capacidades, y en cómo el valor de la dignidad y el desarrollo humano pueden ayudar a superar las limitaciones del actual sistema contractual democrático.

Por último, y dentro de las posibles temáticas afines, dos artículos completan este monográfico. Por un lado, el profesor de la Universidad de Murcia, Manuel Aparicio Payá, se centra en cómo la ética discursiva de K. O. Apel 
(1985) y J. Habermas (2000), propicia un enfoque adecuado para justificar las obligaciones hacia las personas con diversidad funcional. Sin duda, un análisis novedoso dentro de esta teoría ética, que tiene en cuenta, no solo el procedimentalismo kantiano transformado, sino también la ética de la razón cordial de A. Cortina (2009) como estimación de los valores también anclados en la corporalidad. Por otro lado, el artículo de Christian Almonacid Díaz analiza cómo la teoría de la imaginación de P. Ricoeur (1994) no solo se asienta en la literatura, sino que puede contribuir beneficiosamente a la práctica social y a la actividad política, en cuanto entendamos la estructura semiótica de la realidad social.

Como se puede observar a través de la lectura de estos artículos, este monográfico de Recerca. Revista de Pensament i Anàlisi reflexiona y aporta muchas orientaciones sobre la relación actual entre las neurociencias y las ciencias sociales y humanas, tomando la neuroética y la neuroeducación como pilares centrales, pero abordando también conceptos clave como: la interdisciplinariedad, el naturalismo, la metodología neurocientífica, la relación emoción-razón, el papel de las emociones en la vida política y la esfera pública, o la relación entre mente y cerebro. Pero, si algo tienen en común los artículos además de su temática, es que todos apuestan por una mayor perspectiva ética, ya sea en el tratamiento de las neurociencias sociales, como la neuroeducación o la neuropolítica, o en la deliberación pública y en las relaciones con otros seres humanos.

En consecuencia, las neurociencias pueden contribuir valiosamente al desarrollo de las ciencias humanas -y viceversa- siempre y cuando encuentren un método de relación donde se eviten imposiciones de lo empírico sobre lo social, y donde no se obvie la perspectiva filosófica de reflexión sobre la ciencia, para que así esta no tenga un sentido estrecho, sino amplio.

\section{BIBLIOGRAFÍA}

Ansari, Daniel (2005). Time to use neuroscience findings in teacher training. Nature, 437, 26.

Ansari, Daniel, Coch, Donna \& De Smedt, Bert (2011). Connecting Education and Cognitive Neuroscience: Where will the journey take us? Educational Philosophy and Theory, 43(1), 37-42.

Apel, Karl O. (1985b). ¿Límites de la ética discursiva? En Cortina,Adela (ed.). Razón comunicativa y responsabilidad solidaria (233-262). Barcelona: Sígueme. 
Bowers, Jeffrey S. (2016). The practical and principled problems with educational neuroscience. Psychological Review, 123, 600-612.

Campbell, Stephen R (2011). Educational Neuroscience: Motivations, methodology and implications. Educational Philosophy and Theory, 43(1), 7-16. doi: 10.1111/j.1469-5812.2010.00701.x.

Codina, María José (2015). Neuroeducación en virtudes cordiales. Cómo reconciliar lo que decimos con lo que bacemos. Barcelona: Octaedro.

Conill, Jesús (2012). Neuroeconomía y Neuromarketing. ¿Más allá de la racionalidad maximizadora? En Cortina,A. (ed.), Guía Comares de Neurofilosofía Práctica (39-64). Granada: Comares.

Conill, Jesús \& Pérez-Zafrilla, Pedro Jesús (eds) (2013). Retos actuales de la neuroética. Recerca. Revista de Pensament i Anàlisi, 13.

Cortina, Adela (ed.) (2012). Guía Comares de Neurofilosofía Práctica. Granada: Comares.

Cortina, Adela (2009). Ética de la razón cordial. Oviedo: Ediciones Nobel.

Damasio, Antonio (2003). El error de Descartes: La emoción, la razón y el cerebro humano. Barcelona: Editorial Crítica S.L.

Descartes, Renné (1622/2005b). Las pasiones del alma. Madrid: Editorial Edaf, SA.

Evers, Kathinka (2010). Neuroética. Cuando la materia se despierta (Víctor Goldstein, trad.) Madrid: Katz.

Farah, Martha J. (2011). Neuroscience and neuroethics in the 21st century. En Illes, Judy \& Sahakian, Barbara J. (eds.), The Oxford Handbook of Neuroethics (761-781). Oxford: Oxford University Press.

Feenstra, Ramón \& Pallarés-Domínguez, Daniel (2017). Debates éticos en torno al neuromarketing político: el avance tecnológico y su potencial incidencia en la formación de la opinión pública. Veritas, 36, 9-28.

García-Marzá, Domingo (2012a). Neuropolítica: una mirada crítica sobre el neuropoder. En Cortina, Adela (ed.), Guía Comares de Neurofilosofía Práctica (77-96). Granada: Comares.

García-Marzá, Domingo \& Feenstra, Ramón (eds.) (2013). Ética y neurociencias: la aportación a la política, la economía y la educación. Castellón: Servei de Publicacions de la Universitat Jaume I.

Glannon, Walter (2006). Neuroethics. Bioethics, 20(1), 37-52.

Goswami, Usha (2004). Neuroscience and education. British Journal of Educational Psychology, 74, 1-14.

Habermas, Jürgen (2000). Aclaraciones a la ética del discurso. Madrid: Trotta. 
Haidt, Jonathan (2012). The righteous mind. Why good people are divided by politics and religion. Nueva York: Pantheon Books.

Howard-Jones, Paul (2011): Investigación neuroeducativa. Neurociencia, educación y cerebro: de los contextos a la práctica (Pablo Manzano, trad.). Madrid: La Muralla.

Lakoff, George (2009). The political mind. A cognitive scientist guide to your brain and its politics. Nueva York: Penguin Books.

Levy, Neil (2007). Neuroethics. Challenges for the 21st Century. Cambridge: Cambridge University Press.

Nussbaum, Martha Craven (2002). Las mujeres y el desarrollo bumano: el enfoque de las capacidades. Barcelona: Herder.

Ortiz de Landázuri, Carlos (2016). El error neurocientífico de Descartes, entre Spinoza y Aquinas. El debate entre Damasio y Stump sobre el carácter eliminativo o vitalista del materialismo en la neuroética, neuropolítica y neuroeconomía. Recerca. Revista de Pensament i Anàlisi, 18, 107-133.

Ricoeur, Paul (1994). Ideología y utopía. Barcelona: Gedisa.

Roskies, Adina (2002). Neuroethics for the New Millenium. Neuron, 35, 21-23. Taylor, John (1996a). Las fuentes del yo. La construcción de la identidad moderna. Barcelona: Paidós. 